\title{
On Full-State Hybrid Projective Synchronization of General Discrete Chaotic Systems
}

\author{
Adel Ouannas \\ LAMIS Laboratory, Department of Mathematics and Computer Science, University of Tebessa, 12002 Tebessa, Algeria \\ Correspondence should be addressed to Adel Ouannas; ouannas_adel@yahoo.fr
}

Received 28 July 2014; Revised 27 September 2014; Accepted 28 September 2014; Published 14 October 2014

Academic Editor: Ivo Petras

Copyright (C) 2014 Adel Ouannas. This is an open access article distributed under the Creative Commons Attribution License, which permits unrestricted use, distribution, and reproduction in any medium, provided the original work is properly cited.

\begin{abstract}
The problems of full-state hybrid projective synchronization (FSHPS) and inverse full-state hybrid projective synchronization (IFSHPS) for general discrete chaotic systems are investigated in 2D. Based on nonlinear control method and Lyapunov stability theory, new controllers are designed to study FSHPS and IFSHPS, respectively, for 2D arbitrary chaotic systems in discrete-time. Numerical example and simulations are used to validate the main results of this paper.
\end{abstract}

\section{Introduction}

Many mathematical models of biological processes, physical processes, and chemical processes, etc., were dened using chaotic dynamical systems in discrete-time. Recently, more and more attention were paid to chaos synchronization in discrete-time dynamical systems [1-3]. Synchronization of discrete chaotic dynamical systems has also potential applications in secure communication $[4,5]$. Since the work of Pecora and Carroll [6], various powerful methods and techniques have been proposed to investigate chaos synchronization in dynamical systems $[7,8]$ and different types of synchronization have been reported [9-11].

Recently, a novel type of synchronization, known as fullstate hybrid projective synchronization (FSHPS), has been introduced and applied to chaotic systems in continuoustime [12], which includes projective synchronization (PS) and hybrid projective synchronization (HPS). In FSHPS, each response system state synchronizes with a linear combination of drive system states. By the same procedure we can define a new type of synchronization, called inverse fullstate hybrid projective synchronization (IFSHPS), when each drive system state synchronizes with a linear combination of response system states.

In this paper, based on nonlinear control method in 2D and discrete-time Lyapunov stability theory, firstly, a new synchronization controller is designed for full-state hybrid projective synchronization (FSHPS) of general chaotic systems. Secondly, a new control scheme is proposed to study the problem of inverse full-state hybrid projective synchronization (IFSHPS) for arbitrary chaotic systems. The synchronization criterions derived in this paper are established in the form of simple algebraic conditions about the linear part of the response system and the drive system, respectively, which are very convenient to verify. In order to show the effectiveness of the proposed synchronization schemes, our approach is applied to the drive Fold discretetime system and the controlled Lorenz discrete-time system to achieve FSHPS and IFSHPS, respectively.

The remainder of this paper is organized as follows. In Section 2, definitions of FSHPS and IFSHPS are introduced. In Section 3, a new controller is designed to study FSHPS. In Section 4, a new synchronization criterion for IFSHPS is derived. In Section 5, numerical application and simulations are given to show the use of the proposed control schemes and the derived synchronization criterions. Finally, conclusion is followed in Section 6.

\section{Definitions of FSHPS and IFSHPS}

Consider the following coupled chaotic systems:

$$
\begin{aligned}
x_{i}(k+1) & =f_{i}(X(k)), \\
y_{i}(k+1) & =g_{i}(Y(k))+u_{i}, \\
1 & \leq i \leq n,
\end{aligned}
$$


where $X(k)=\left(x_{i}(k)\right)_{1 \leq i \leq n}, Y(k)=\left(y_{i}(k)\right)_{1 \leq i \leq n}$ are the state vectors of drive system and response system, respectively, $f_{i}$ : $\mathbb{R}^{n} \rightarrow \mathbb{R}, g_{i}: \mathbb{R}^{n} \rightarrow \mathbb{R},(1 \leq i \leq n)$, and $u_{i},(1 \leq i \leq n)$, are controllers.

We present the definition of full-state hybrid projective synchronization (FSHPS) for the drive-response chaotic systems (1).

Definition 1. The coupled drive-response chaotic systems (1) are in full-state hybrid projective synchronization (FSHPS) when, for an initial condition, there exist controllers $u_{i},(1 \leq$ $i \leq n)$, and given real constants $\left(\theta_{i j}\right) \in \mathbb{R}^{n \times n}$ such that the synchronization errors

$$
e_{i}(k)=y_{i}(k)-\sum_{i=1}^{n} \theta_{i j} x_{j}(k), \quad 1 \leq i \leq n,
$$

satisfy that $\lim _{k \rightarrow \infty} e_{i}(k)=0$ for $i=1,2, \ldots, n$.

The definition of inverse full-state hybrid projective synchronization (IFSHPS) is given next.

Definition 2. The coupled drive-response chaotic systems (1) are in inverse full-state hybrid projective synchronization (IFSHPS) when, for an initial condition, there exist controllers $\left(u_{i}\right)_{1 \leq i \leq n}$ and given real constant $\left(\theta_{i j}\right) \in \mathbb{R}^{n \times n}$ such that the synchronization errors

$$
e_{i}(k)=x_{i}(k)-\sum_{i=1}^{n} \theta_{i j} y_{j}(k), \quad 1 \leq i \leq n,
$$

satisfy that $\lim _{k \rightarrow \infty} e_{i}(k)=0$ for $i=1,2, \ldots, n$.

\section{Controller Design for FSHPS in 2D}

In this section, we consider the drive system in the following form:

$$
\begin{aligned}
& x_{1}(k+1)=f_{1}(X(k)), \\
& x_{2}(k+1)=f_{2}(X(k)),
\end{aligned}
$$

where $X(k)=\left(x_{1}(k), x_{2}(k)\right)^{T}$ is the state vector of the drive system (4), $f_{i}: \mathbb{R}^{2} \rightarrow \mathbb{R}(i=1,2)$.

As the response system, we consider the following chaotic system:

$$
\begin{aligned}
& y_{1}(k+1)=\sum_{j=1}^{2} b_{1 j} y_{j}(k)+g_{1}(Y(k))+u_{1}, \\
& y_{2}(k+1)=\sum_{j=1}^{2} b_{2 j} y_{j}(k)+g_{2}(Y(k))+u_{2},
\end{aligned}
$$

where $Y(k)=\left(y_{1}(k), y_{2}(k)\right)^{T}$ is the state vector of the response system (5), $\left(b_{i j}\right) \in \mathbb{R}^{2 \times 2}, g_{i}: \mathbb{R}^{2} \rightarrow \mathbb{R}(i=1,2)$, are nonlinear functions, and $\left(u_{1}, u_{2}\right)^{T}$ is the vector controller.
According to the definition of FSHPS, the synchronization errors between the drive system (4) and the response system (5) are defined as

$$
\begin{aligned}
& e_{1}(k+1)=y_{1}(k+1)-\theta_{11} x_{1}(k+1)-\theta_{12} x_{2}(k+1), \\
& e_{2}(k+1)=y_{2}(k+1)-\theta_{21} x_{1}(k+1)-\theta_{22} x_{2}(k+1),
\end{aligned}
$$

where $\left(\theta_{i j}\right) \in \mathbb{R}^{2 \times 2}$ are arbitrary scaling constants.

Then, the synchronization errors of FSHPS between systems (4) and (5) can be derived as

$$
\begin{aligned}
& e_{1}(k+1)=\sum_{j=1}^{2} b_{1 j} e_{j}(k)+L_{1}+N_{1}+u_{1}, \\
& e_{2}(k+1)=\sum_{j=1}^{2} b_{2 j} e_{j}(k)+L_{2}+N_{2}+u_{2},
\end{aligned}
$$

where

$$
\begin{aligned}
& L_{1}=\sum_{j=1}^{2} \lambda_{1 j} x_{j}(k), \\
& L_{2}=\sum_{j=1}^{2} \lambda_{2 j} x_{j}(k),
\end{aligned}
$$

where

$$
\begin{aligned}
& \lambda_{11}=b_{11} \theta_{11}+b_{12} \theta_{21}, \\
& \lambda_{12}=b_{11} \theta_{12}+b_{12} \theta_{22}, \\
& \lambda_{21}=b_{21} \theta_{11}+b_{22} \theta_{21}, \\
& \lambda_{22}=b_{21} \theta_{12}+b_{22} \theta_{22}, \\
& N_{1}=g_{1}-\theta_{11} f_{1}-\theta_{12} f_{2}, \\
& N_{2}=g_{2}-\theta_{21} f_{1}-\theta_{22} f_{2} .
\end{aligned}
$$

To achieve FSHPS between systems (4) and (5), we choose the synchronization controllers as

$$
\begin{aligned}
u_{1}= & \left(b_{22}-l_{1}\right) e_{1}(k)+\left(b_{21}-l_{2}\right) e_{2}(k)-L_{1}-N_{1}, \\
u_{2}= & \left(b_{12}-l_{2}\right) e_{1}(k)-\left(2 b_{22}+b_{11}-l_{2}\right) e_{2}(k) \\
& -L_{2}-N_{2},
\end{aligned}
$$

where $l_{1}$ and $l_{2}$ are control constants to be determined.

Theorem 3. If the control constants $\left(l_{i}\right)_{1 \leq i \leq 2}$ are chosen such that

$$
\left(b_{11}+b_{22}-l_{1}\right)^{2}+\left(b_{21}+b_{12}-l_{2}\right)^{2}<1,
$$

then the drive system (4) and the response (5) are globally fullstate hybrid projective synchronized. 
Proof. By substituting the control law (10) into (7), the synchronization errors can be written as

$$
\begin{aligned}
e_{1}(k+1)= & \left(b_{11}+b_{22}-l_{1}\right) e_{1}(k) \\
& +\left(b_{12}+b_{21}-l_{2}\right) e_{2}(k), \\
e_{2}(k+1)= & \left(b_{21}+b_{12}-l_{2}\right) e_{1}(k) \\
& -\left(b_{22}+b_{11}-l_{1}\right) e_{2}(k) .
\end{aligned}
$$

Construct the candidate Lyapunov function in the form

$$
V(e(k))=e_{1}^{2}(k)+e_{2}^{2}(k)
$$

we get

$$
\begin{aligned}
\Delta V & (e(k)) \\
= & V(e(k+1))-V(e(k)) \\
= & \sum_{i=1}^{2} e_{i}^{2}(k+1)-\sum_{i=1}^{2} e_{i}^{2}(k) \\
= & \left(\left(b_{11}+b_{22}-l_{1}\right)^{2}+\left(b_{21}+b_{12}-l_{2}\right)^{2}-1\right) \\
& \cdot\left(e_{1}^{2}(k)+e_{2}^{2}(k)\right) \\
& +2\left[\left(b_{11}+b_{22}-l_{1}\right)\left(b_{12}+b_{22}-l_{2}\right)\right. \\
& \left.\quad-\left(b_{21}+b_{12}-l_{2}\right)\left(b_{11}+b_{22}-l_{1}\right)\right] \\
& \cdot e_{1}(k) e_{2}(k) .
\end{aligned}
$$

By using (11), we obtain $\Delta V(e(k))<0$. Thus, by Lyapunov stability theory, it is immediate that $\lim _{k \rightarrow \infty} e_{i}(k)=$ $0(i=1,2)$, and we conclude that the two systems (4) and (5) are globally full-state hybrid projective synchronized.

\section{New Criterion for IFSHPS in 2D}

Now, we consider the drive and the response chaotic systems are in the following forms:

$$
\begin{aligned}
& x_{i}(k+1)=\sum_{j=1}^{2} a_{i j} x_{j}(k)+f_{i}(X(k)), \quad 1 \leq i \leq 2, \\
& y_{i}(k+1)=g_{i}(Y(k))+u_{i}, \quad 1 \leq i \leq 2,
\end{aligned}
$$

where $X(k)=\left(x_{1}(k), x_{2}(k)\right)^{T}, Y(k)=\left(y_{1}(k), y_{2}(k)\right)^{T}$ are the state vectors of drive system and the response systems, respectively, $\left(a_{i j}\right) \in \mathbb{R}^{2 \times 2}, f_{i}: \mathbb{R}^{2} \rightarrow \mathbb{R}(i=1,2)$, are nonlinear functions, $g_{i}: \mathbb{R}^{2} \rightarrow \mathbb{R}(i=1,2)$, and $\left(u_{i}\right)_{1 \leq i \leq 2}$ are synchronization controllers.

According to the definition of IFSHPS, the synchronization errors between the drive system (15) and the response system (16) are defined as

$$
\begin{aligned}
& e_{1}(k+1)=x_{1}(k+1)-\theta_{11} y_{1}(k+1)-\theta_{12} y_{2}(k+1), \\
& e_{2}(k+1)=x_{2}(k+1)-\theta_{21} y_{1}(k+1)-\theta_{22} y_{2}(k+1),
\end{aligned}
$$

Then, the synchronization errors of IFSHPS between systems (15) and (16) can be derived as

$$
\begin{aligned}
e_{1}(k+1)= & \left(a_{21}-l_{1}\right) e_{1}(k)-\left(a_{22}+2 a_{12}-l_{2}\right) e_{2}(k) \\
& +R_{1}-\sum_{j=1}^{2} \theta_{1 j} u_{j}, \\
e_{2}(k+1)= & \left(a_{11}-l_{1}\right) e_{1}(k)+\left(a_{12}-l_{2}\right) e_{2}(k)+R_{2} \\
& -\sum_{j=1}^{2} \theta_{2 j} u_{j},
\end{aligned}
$$

where $\left(l_{i}\right)_{1 \leq i \leq 2}$ are control constants and

$$
\begin{aligned}
& R_{1}=\sum_{j=1}^{2} \lambda_{1 j} y_{j}(k)+f_{1}-\theta_{11} g_{1}-\theta_{12} g_{2}, \\
& R_{2}=\sum_{j=1}^{2} \lambda_{2 j} y_{j}(k)+f_{2}-\theta_{21} g_{1}-\theta_{22} g_{2},
\end{aligned}
$$

where

$$
\begin{aligned}
& \lambda_{11}=a_{11} \theta_{11}+a_{12} \theta_{21}, \\
& \lambda_{12}=a_{11} \theta_{12}+a_{12} \theta_{22}, \\
& \lambda_{21}=a_{21} \theta_{11}+a_{22} \theta_{21}, \\
& \lambda_{22}=a_{21} \theta_{12}+a_{22} \theta_{22} .
\end{aligned}
$$

To achieve IFSHPS between systems (15) and (16), we assume that

$$
\theta_{11} \theta_{22} \neq \theta_{12} \theta_{21}
$$

and we choose the synchronization controllers as

$$
\begin{aligned}
& u_{1}=\frac{\theta_{22} R_{1}-\theta_{12} R_{2}}{\theta_{12} \theta_{21}-\theta_{11} \theta_{22}}, \\
& u_{2}=\frac{\theta_{11} R_{2}-\theta_{21} R_{1}}{\theta_{12} \theta_{21}-\theta_{11} \theta_{22}} .
\end{aligned}
$$

Theorem 4. If the control constants $\left(l_{i}\right)_{1 \leq i \leq 2}$ are chosen such that

$$
\begin{aligned}
& \left|a_{11}+a_{21}-l_{1}\right|<\frac{1}{\sqrt{2}}, \\
& \left|a_{22}+a_{12}-l_{2}\right|<\frac{1}{\sqrt{2}},
\end{aligned}
$$

then the drive system (15) and the response (16) are globally inverse full-state hybrid projective synchronized.

Proof. By substituting control law (22) into (18), the synchronization errors can be written as

$$
\begin{aligned}
e_{1}(k+1)= & \left(a_{11}+a_{21}-l_{1}\right) e_{1}(k) \\
& -\left(a_{12}+a_{22}-l_{2}\right) e_{2}(k), \\
e_{2}(k+1)= & \left(a_{21}+a_{11}-l_{1}\right) e_{1}(k) \\
& +\left(a_{22}+a_{12}-l_{2}\right) e_{2}(k) .
\end{aligned}
$$

where $\left(\theta_{i j}\right) \in \mathbb{R}^{2 \times 2}$ are scaling constants. 
Construct the candidate Lyapunov function in the form

$$
V(e(k))=e_{1}^{2}(k)+e_{2}^{2}(k)
$$

we get

$$
\begin{aligned}
\Delta V & (e(k)) \\
= & V(e(k+1))-V(e(k)) \\
= & \sum_{i=1}^{2} e_{i}^{2}(k+1)-\sum_{i=1}^{2} e_{i}^{2}(k) \\
= & \left(2\left(a_{11}+a_{21}-l_{1}\right)^{2}-1\right) e_{1}^{2}(k) \\
& +\left(2\left(a_{22}+a_{12}-l_{2}\right)^{2}-1\right) e_{1}^{2}(k) \\
& +2\left[\left(a_{11}+a_{21}-l_{1}\right)\left(a_{22}+a_{12}-l_{2}\right)\right. \\
& \left.\quad-\left(a_{11}+a_{21}-l_{1}\right)\left(a_{22}+a_{12}-l_{2}\right)\right] \\
& \cdot e_{1}(k) e_{2}(k) .
\end{aligned}
$$

By using (23), we obtain $\Delta V(e(k))<0$. Thus, by Lyapunov stability theory, it is immediate that $\lim _{k \rightarrow \infty} e_{i}(k)=$ $0(i=1,2)$, and we conclude that the two systems (15) and (16) are globally inverse full-state hybrid projective synchronized.

\section{Numerical Application and Simulations}

We consider the discrete-time Fold system system as the drive system and the controlled Lorenz discrete-time as the response system. The discrete-time Fold system can be described as

$$
\begin{aligned}
& x_{1}(k+1)=\alpha x_{1}(k)+x_{2}(k), \\
& x_{2}(k+1)=x_{1}^{2}(k)+\beta,
\end{aligned}
$$

which has a chaotic attractor, for example, when $(\alpha, \beta)=$ $(-0.1,-1.7)[13]$. The discrete-time Fold system is shown in Figure 1.

The controlled Lorenz discrete-time system can be described as

$$
\begin{aligned}
& y_{1}(k+1)=(1+a b) y_{1}(k)-b y_{1}(k) y_{2}(k)+u_{1}, \\
& y_{2}(k+1)=(1-b) y_{2}(k)+b y_{1}^{2}(k)+u_{2},
\end{aligned}
$$

where $U=\left(u_{1}, u_{2}\right)^{T}$ is the vector controller. The Lorenz discrete-time has a chaotic attractor, for example, when $(a, b)=(1.25,0.75)[13]$. The chaotic attractor of the Lorenz discrete-time system is shown in Figure 2.

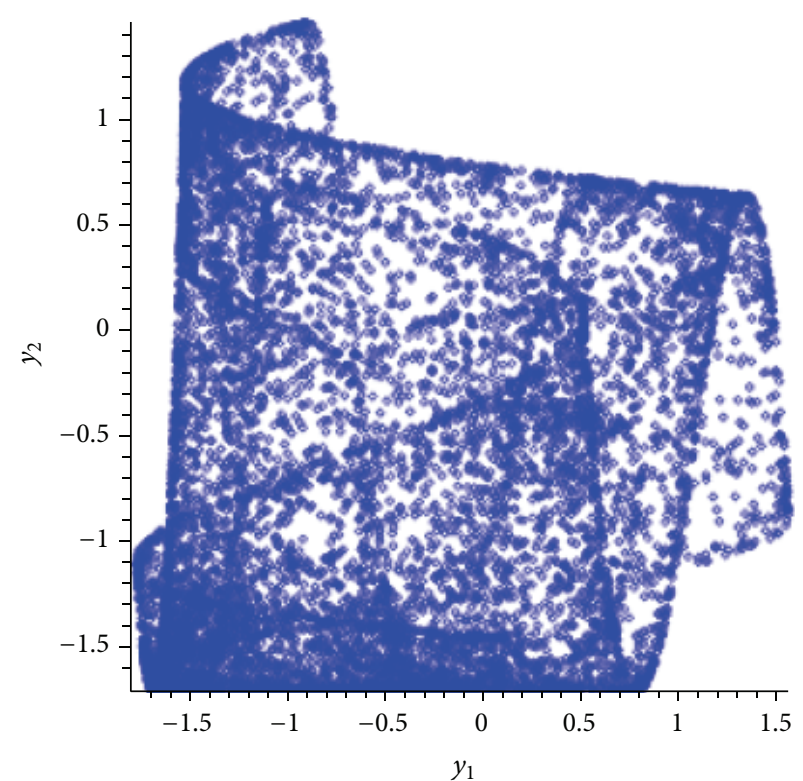

FIGURE 1: Chaotic attractor of Fold discrete-time system when $(\alpha, \beta)=(-0.1,-1.7)$.

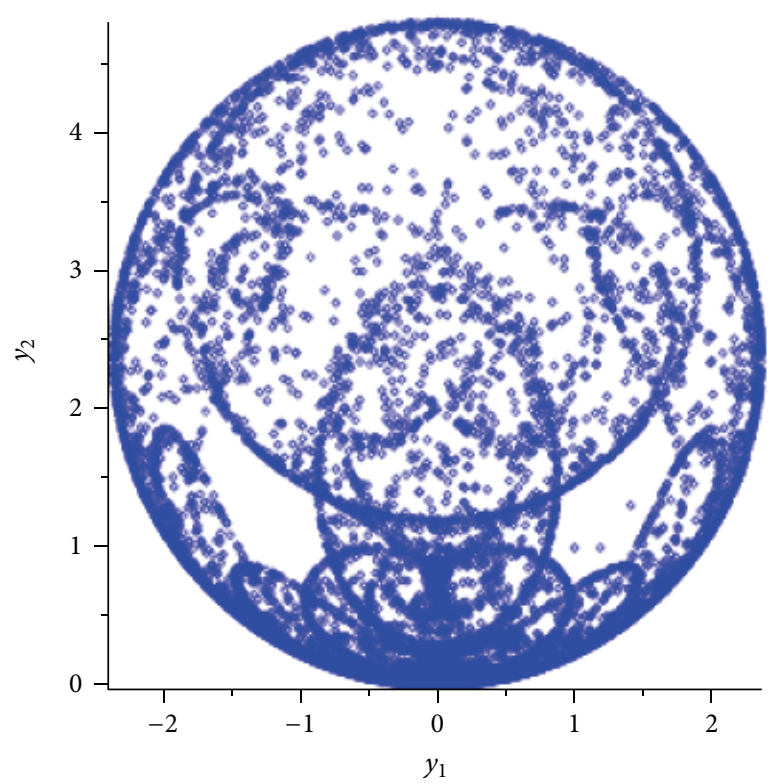

FIgURE 2: Chaotic attractor of Lorenz discrete-time system when $(a, b)=(1.25,0.75)$.

5.1. FSHPS between the Discrete-Time Fold System and the Controlled Lorenz Discrete-Time System. Her, according to our approach presented in Section 3, we obtain

$$
\begin{aligned}
& \left(\begin{array}{ll}
b_{11} & b_{12} \\
b_{21} & b_{22}
\end{array}\right)=\left(\begin{array}{cc}
1+a b & 0 \\
0 & 1-b
\end{array}\right), \\
& e_{1}(k+1)=\left[2+(a-1) b-l_{1}\right] e_{1}(k)-l_{2} e_{2}(k), \\
& e_{2}(k+1)=-l_{2} e_{1}(k)-\left[2+(a-1) b-l_{1}\right] e_{2}(k) .
\end{aligned}
$$




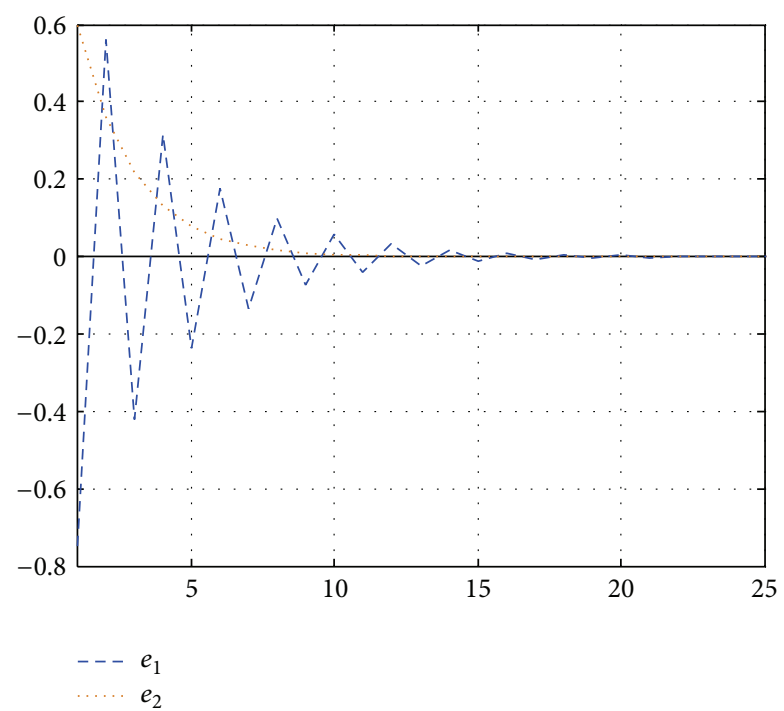

FIGURE 3: Time evolution of FSHPS errors between the drive discrete-time Fold system (27) and the controlled Lorenz discretetime system (28).

Corollary 5. The drive Fold discrete-time system and the response Lorenz discrete-time system are globally full-state hybrid projective synchronized, if the control constants are chosen such that

$$
\left(2.18-l_{1}\right)^{2}+l_{2}^{2}<1 .
$$

If we take $\left(l_{1}, l_{2}\right)=(2,0.5)$ and by using Matlab, we get the numeric result that is shown in Figure 3.

\subsection{Inverse FSHPS between the Discrete-Time Fold System and} the Controlled Lorenz Discrete-Time System. Now, according to our approach presented in Section 4, we obtain

$$
\begin{aligned}
\left(\begin{array}{ll}
a_{11} & a_{12} \\
a_{21} & a_{22}
\end{array}\right) & =\left(\begin{array}{ll}
\alpha & 1 \\
0 & 0
\end{array}\right), \\
e_{1}(k+1) & =\left(\alpha-l_{1}\right) e_{1}(k)-\left(1-l_{2}\right) e_{2}(k), \\
e_{2}(k+1) & =\left(\alpha-l_{1}\right) e_{1}(k)+\left(1-l_{2}\right) e_{2}(k) .
\end{aligned}
$$

Corollary 6. The drive Fold discrete-time system and the response Lorenz discrete-time system are globally inverse fullstate hybrid projective synchronized, if the control constants are chosen such that

$$
\begin{array}{r}
-0.8<l_{1}<0.6, \\
0.3<l_{2}<1.7 .
\end{array}
$$

Finally, if we take $\left(l_{1}, l_{2}\right)=(0.5,1)$ and by using Matlab, we get the numeric result that is shown in Figure 4.

\section{Conclusion}

In this paper, to study FSHPS and IFSHPS between arbitrary chaotic dynamical systems in 2D discrete-time, a new

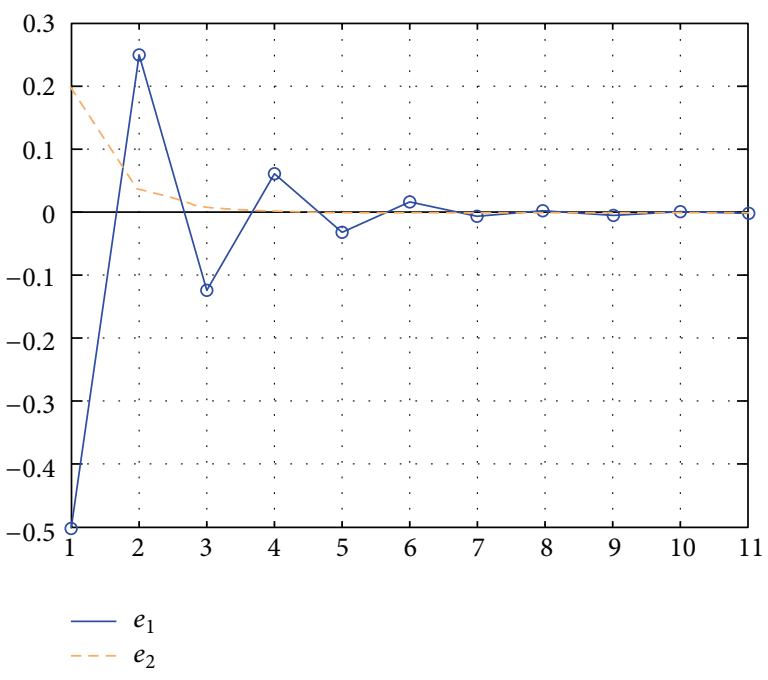

FIGURE 4: Time evolution of inverse FSHPS errors between the drive discrete-time Fold system (27) and the controlled Lorenz discretetime system (28).

nonlinear control method was presented. It was shown that the proposed synchronization criterions were based on simple and effective results. Firstly, FSHPS is achieved by controlling the linear part of the response system. Secondly, IFSHPS is guaranteed when the linear part of the drive system is controlled. Finally, numerical example and simulations results were utilized to illustrate the effectiveness of the proposed schemes.

\section{Conflict of Interests}

The authors declare that there is no conflict of interests regarding the publication of this paper.

\section{References}

[1] W. Liu, Z. M. Wang, and W. D. Zhang, "Controlled synchronization of discrete-time chaotic systems under communication constraints," Nonlinear Dynamics, vol. 69, no. 1-2, pp. 223-230, 2012.

[2] G. Grassi, "Arbitrary full-state hybrid projective synchronization for chaotic discrete-time systems via a scalar signal," Chinese Physics B, vol. 21, no. 6, Article ID 060504, 2012.

[3] Z. Yan, "Q-S synchronization in 3D Hénon-like map and generalized Hénon map via a scalar controller," Physics Letters A, vol. 342, no. 4, pp. 309-317, 2005.

[4] J. G. Lu and Y. G. Xi, "Chaos communication based on synchronization of discrete-time chaotic systems," Chinese Physics, vol. 14, no. 2, pp. 274-278, 2005.

[5] E. Solak, "Cryptanalysis of observer based discrete-time chaotic encryption schemes," International Journal of Bifurcation and Chaos in Applied Sciences and Engineering, vol. 15, no. 2, pp. 653658, 2005.

[6] L. M. Pecora and T. L. Carroll, "Synchronization in chaotic systems," Physical Review Letters, vol. 64, no. 8, pp. 821-824, 1990. 
[7] W. Xiao-Qun and L. Jun-An, "Parameter identification and backstepping control of uncertain Lü system," Chaos, Solitons and Fractals, vol. 18, no. 4, pp. 721-729, 2003.

[8] C.-C. Yang and C.-L. Lin, "Robust adaptive sliding mode control for synchronization of space-clamped FitzHugh-Nagumo neurons," Nonlinear Dynamics, vol. 69, no. 4, pp. 2089-2096, 2012.

[9] T. Banerjee, D. Biswas, and B. C. Sarkar, "Complete and generalized synchronization of chaos and hyperchaos in a coupled first-order time-delayed system," Nonlinear Dynamics, vol. 71, no. 1-2, pp. 279-290, 2013.

[10] M. M. Al-Sawalha and M. S. M. Noorani, "Anti-synchronization between two different hyperchaotic systems," Journal of Uncertain Systems, vol. 3, no. 3, pp. 192-200, 2009.

[11] A. Khan and R. P. Prasad, "Projective synchronization of different hyper-chaotic systems by active nonlinear control," Journal of Uncertain Systems, vol. 8, no. 2, pp. 90-100, 2014.

[12] M. Hu, Z. Xu, and R. Zhang, "Full state hybrid projective synchronization in continuous-time chaotic (hyperchaotic) systems," Communications in Nonlinear Science and Numerical Simulation, vol. 13, no. 2, pp. 456-464, 2008.

[13] Z. Yan, "Q-S (complete or anticipated) synchronization backstepping scheme in a class of discrete-time chaotic (hyperchaotic) systems: a symbolic-numeric computation approach," Chaos, vol. 16, no. 1, Article ID 013119, 11 pages, 2006. 

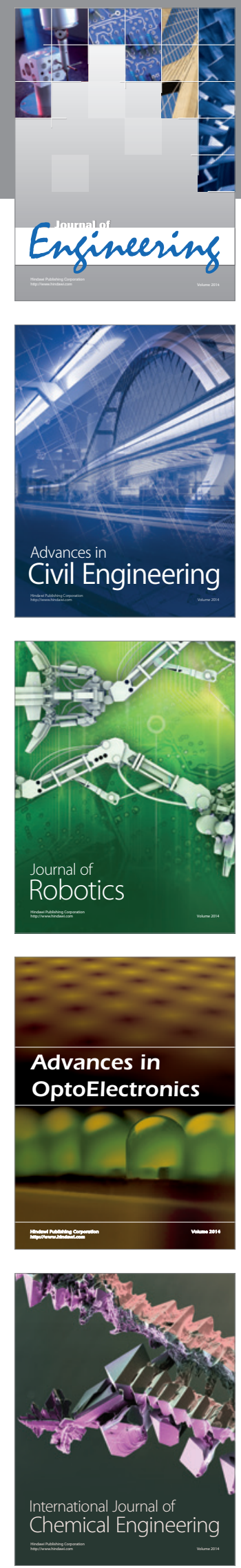

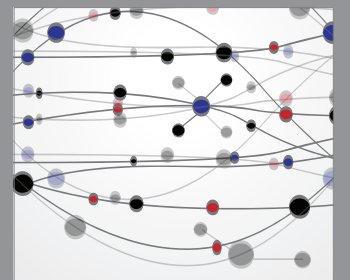

The Scientific World Journal
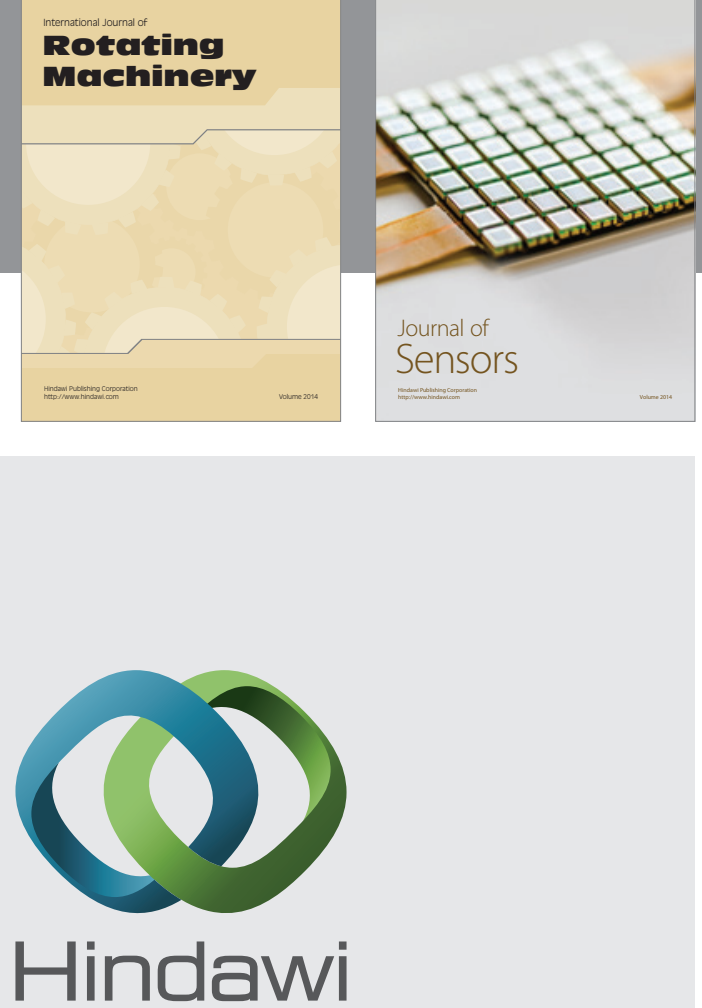

Submit your manuscripts at http://www.hindawi.com
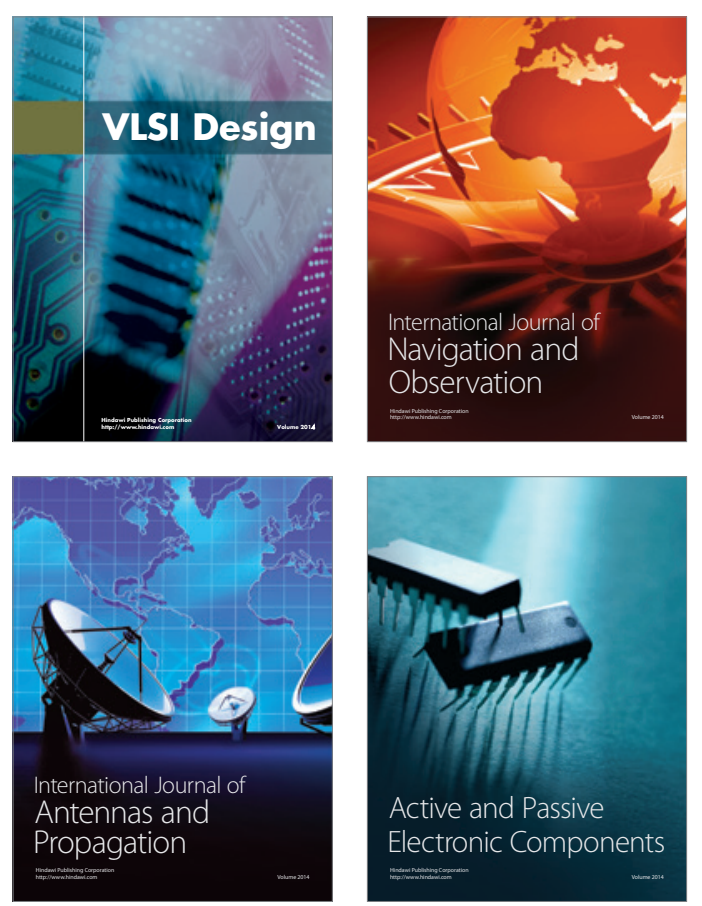
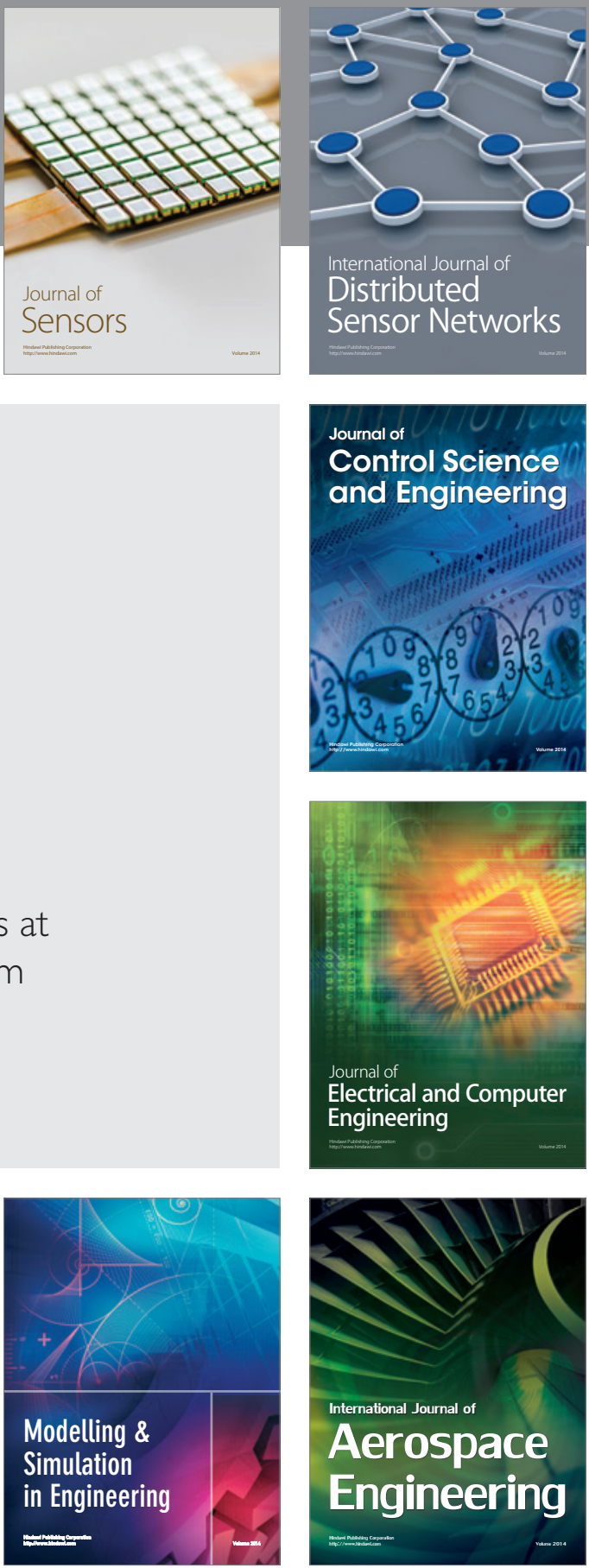

Journal of

Control Science

and Engineering
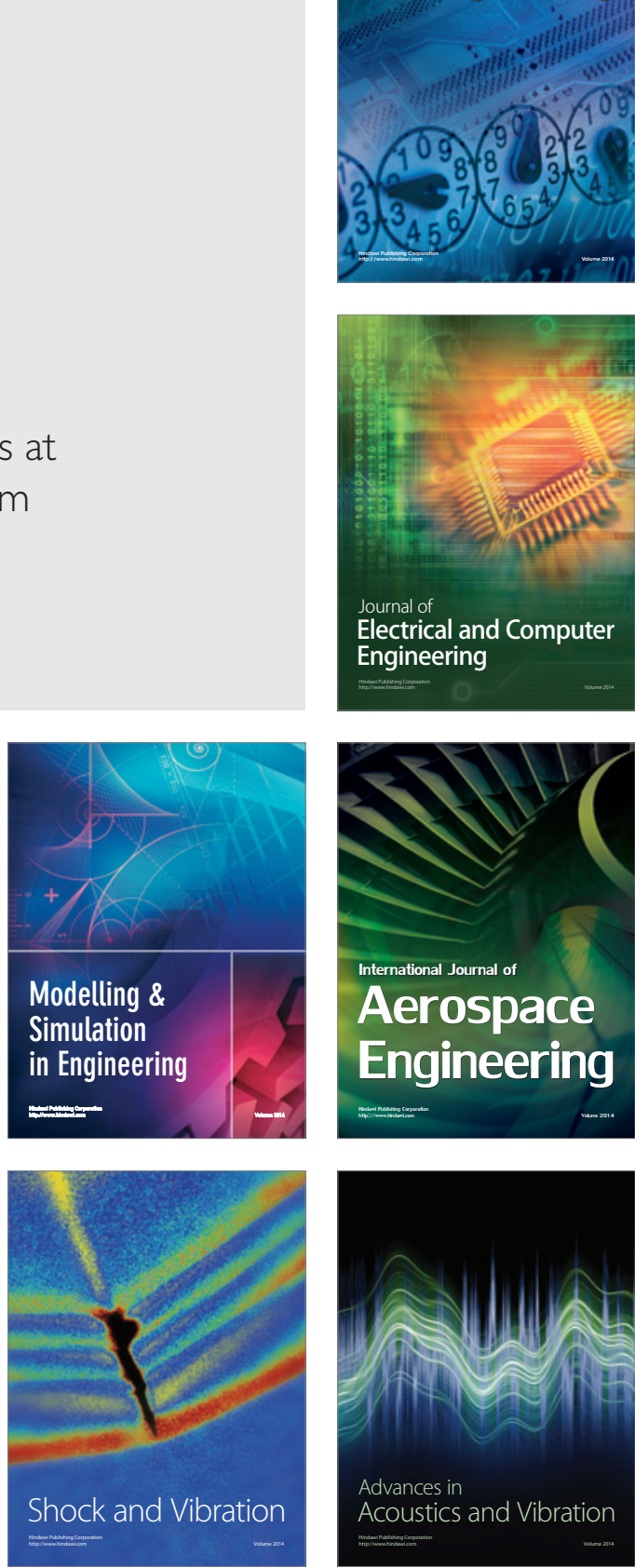